\title{
Research Status and Prospect of Blockchain Technology in Agriculture Field
}

\author{
Dawei $\mathrm{Xu}^{1,2(\bowtie)}$, Weiqi Wang ${ }^{2}$, Liehuang $\mathrm{Zhu}^{1}$, and Ruiguang $\mathrm{Li}^{2,3}$ \\ ${ }^{1}$ School of Computer Science and Technology, Beijing Institute of Technology, Beijing, China \\ 3220195131@bit.edu.cn \\ 2 College of Cybersecurity, Changchun University, Changchun, China \\ 3 National Computer Network Emergency Response Technical Team/Coordination Center \\ of China, Beijing, China
}

\begin{abstract}
Agriculture 4.0 is the era of integrating intelligent technologies in agriculture. Problems such as low informatization, food safety, high management cost and imbalance between supply and demand in agriculture have greatly hindered the development of agriculture. The various properties of blockchain technology can make up for the lack of agricultural mechanism, and the fusion of the two is a hot issue in the application of blockchain. Blockchain technology has already had some application cases in the field of agriculture. Based on the research status of Chinese and foreign scholars in this field, this paper firstly introduces the basic overview of blockchain. Then, with agricultural supply chain and agricultural product traceability as the core, it describes the application of blockchain technology in the agricultural field, and further explores solutions to different application problems. Finally, combined with the practical application of "agriculture + blockchain", relevant Suggestions are proposed to provide reference for cross-disciplinary research.
\end{abstract}

Keywords: Blockchain $\cdot$ Agriculture $\cdot$ Supply chain $\cdot$ Tracing

\section{Introduction}

Blockchain technology is considered as the key technology leading intelligent communication and information sharing, is also a hot research field in the current academic circle with the topics mainly focusing on technical basis, security analysis and scenario application.

Agriculture is one of the most important fields in the world. However, the development of agriculture is restricted by its weak foundation, high cost, low efficiency and difficult management. In recent years, to solve the problems existing in the field of agriculture, the research of applying blockchain technology to it has been increasing gradually. In blockchain articles, there are many reviews on the nature of technology, and a few reviews the typical application [1]. This paper first introduces the basic overview of blockchain; Then, with agricultural supply chain and agricultural product traceability as the core, describes the research status and development of blockchain technology in 
the agricultural field, and further explores solutions to different application problems; Finally, combined with the practical application of "agriculture + block chain" to put forward relevant suggestions.

\section{Overview of Blockchain}

\subsection{Core of Blockchain Technology in Agriculture}

The data layer uses hash pointer and follows a certain time sequence to arrange each block consisting of head and body into a chain structure. Each new block needs to pass the consensus verification of $51 \%$ nodes on the chain and load the current data status into the state library. Encryption technology is required to provide privacy protection in the blockchain, and the most representative ones are public key cryptography and hashing algorithm.

Consensus mechanism is the key to determining which miners have the right to generate new blocks. Since it does not rely on the centralized institutions, consensus algorithm is needed as the basis for judgment. This algorithm includes: POW, POS, DPOS, POA, and PBFT. Smart contract of the control layer is the key to the blockchain. It executes the corresponding code program through the computer. It's a script that can self-guarantee and execute a contract without the participation of a third party and geographical restrictions.

\subsection{Application of Blockchain in Agriculture}

The blockchain technology applying in the agriculture field, which point-to-point implementation of transparent transactions are conducive to the collection of agricultural data; Distributed ledger of blockchain enables all participating nodes to share and store unclassified data synchronously, which solves the problem that information cannot be received in real-time among multiple processes; Under the framework of blockchain, the behaviors of participants in the agricultural chain are encouraged and restricted to increase the authenticity of data. In this paper, the research status and development of blockchain in the field of agricultural supply chain and the traceability of agricultural products are mainly described, solutions to different application problems are further explored and relevant suggestions are proposed.

\section{Current Situation of Agricultural Supply Chain}

Current production, supply and marketing in agricultural model is a linear from the producers to the retailers, shown in Fig. 1. Among them, the upstream contains crops and the use of agricultural machinery supervision, middle includes packaging agricultural products processing, cargo transportation, warehousing and logistics, and the downstream contains e-commerce sales and entity. Through trade connected all links, during the transaction should be familiar with the actual needs of customers, passed by quality qualification, provided appropriate payment guarantee. Still many problems in the agricultural supply chain in reality, which fail to meet the conditions that each link should have. Can used to integrate the upper, middle, lower and trading links of the agricultural supply chain into the blockchain, so as to improve the trouble. 


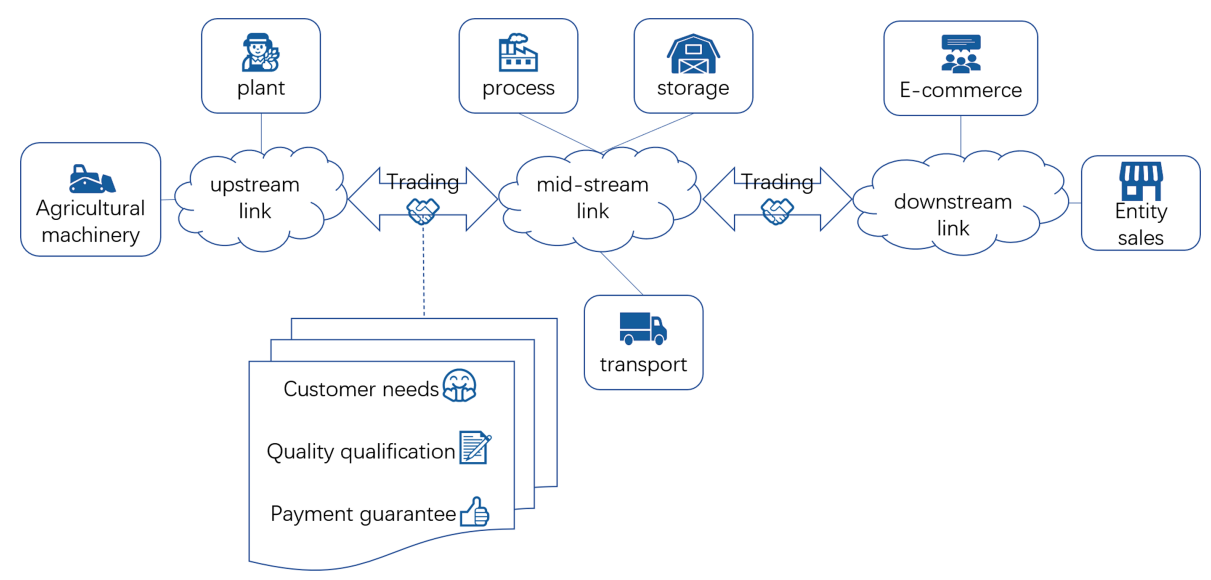

Fig. 1. Agricultural supply chain model

\subsection{Upstream Link}

The upstream link, also called the production link, which includes all the agricultural activities carried out in the farm.

\subsubsection{Agricultural Greenhouse Technology}

Agricultural greenhouse technology is a technology to improve crop yield by controlling environmental factors and is also the key to agricultural production. Patil et al. [2] provide a security framework combining private chain with IoT devices, to provide a secure monitoring communication platform for intelligent greenhouse cultivation to ensure safe communication of devices. Authentication and access control at the physical layer using the immutability of the maintenance ledger; At the communication layer, distributed structures and consensus mechanisms are used to ensure transmission security; There are timestamps and signatures at the data layer to maintain the data authenticity; The interface layer takes the anonymity of the blockchain to solve the changing of attacker's ID.

\subsubsection{Breeding Information Management Technology}

After data collection, store massive data efficiently and safely is more difficult, which puts forward higher requirements for breeding information management technology, and it is necessary to ensure its safety.

Zhang et al. [3] using improved lightweight block chain technology update GSBCP platform software, it covers the whole breeding process from breeding materials to field layout and data collection and analysis. SACBDIBT's storage structure was established, data were divided and stored in multiple databases according to breeding process and location, and summary information was stored in blockchain. When accessing breeding data, system reasonably allocates computing resources and storage space, provides the most idles server as the main server, then encrypts and saves the information in the block chain to improve the security of data. 


\subsubsection{Risk Control Technology}

Technology cannot withstand the damage of various natural disasters to crops. Due to the weak risk resistance of agriculture itself, the existing risk control technology is not perfect, once the damage is accompanied by huge losses. When the database shows that rainfall in farmer's area is below the insured threshold, Kim et al. [4] keeps costs low by using smart contracts to automatically process claims.

\subsection{Mid-Stream Link}

The middle link is related to many users and transactions. The required information includes correct information of agricultural products, processing history of each node to control the production process, etc., which puts forward higher requirements for logistics management technology and quality detection technology.

\subsubsection{Logistics Management Technology}

Logistics help enterprises to realize the whole business effectiveness of the supply chain, is important to the middle link. As the existing logistics management cannot meet the flexibility and efficiency required by the enterprise supply chain, Private Chain or consortium chain can be used to protect personal data, and the validity of data can be maintained by consensus mechanism and intelligent contract. Li Xiaoping et al. [5] took consortium chain as the underlying technology to build LSSC platform, provided consistent interface program for unified format definition of operational data, to realize the assumption of intelligent monitoring and real-time information sharing of agricultural products.

\subsubsection{Quality Inspection Technology}

Quality inspection technology is an important measurement technology in the process of agricultural goods transportation. The transparency of existing technologies fails to satisfy people's needs. The authentication function and non-tampering of blockchain are used to ensure the authenticity of information provided.

Lucena et al. [6] using Hyperledger tracking the source of the delivery batch and establish the special communication channel, the contract has a separate Node. Js process as open application program interface of the business, by Passport. Js configuration of open source authentication middleware access security protection. The results demonstrate that the blockchain technology meets the potential demand for certification, and is expected to increase the value of goods, as real-time sharing reduces disputes and information asymmetry between supply chains.

\subsection{Downstream Link}

The downstream link, also called the sales link, is the process of currency value exchange between agricultural products and users. The e-commerce involved in this link is faced with the problem of information security and sharing, and the efficient flow of resources is difficult. Therefore, blockchain technology is utilized to adjust. 
Huang Wei et al. [7] electricity in rural areas and the value chain of logistics enterprise to carry on the conformity and reconstruction, through the blockchain, dynamic laser anti-counterfeit labels and dynamic image recognition technology to build information tracking and anti-fake model, with the smart contracts to improve the level of automation and prove the authenticity of the product itself and its flow, build public distributed mutualism mode, prompt information safe, efficient, reliable delivery, receipt and payment to ensure electrical business, logistics and customer benefit maximization.

\subsection{Trading Link}

As a bridge between production and sales, the trading link needs to investigate market information, predict customer demand and changes in advance, and communicate with producers in real time, to achieve the balance between supply and demand.

HuoHong et al. [8] with integrated supply chain perspective, with the public, private, consortium chain, a three-state cycle of product, information and economic benefits is formed in the system. Consensus and through the permissions assigned to protect privacy, simultaneously clear regulatory subject realized the agricultural product quality traceability, without intermediary participation, consumer can be directly to produce feedback, taste, quality requirements. Optimize the interest demands of different subjects, ensure the quality, and coordinate the supervision cost and benefit distribution.

\section{Current Status of Agricultural Products Traceability}

The traceability system of agricultural products is mainly responsible for tracking the quality and safety of agricultural products from production to consumption. Its framework is shown in Fig. 2. Agricultural products go through multiple transfers before consumption. To accurately identify the quality of agricultural products, effective detection and prevention of product safety problems and accountability, establishing a reliable traceability system on blockchain is essential.

\subsection{Solves the Problem of Accountability for Agricultural Products}

Due to multiple transfers, people cannot determine the accuracy of information, and the retail industry and enterprises become the most responsible persons. To solve such problems, scholars have been exploring with the help of blockchain and IoT.

Liu Zongmei [9] used consortium chain to build a food traceability platform of "blockchain +RFID", formulated a list of violations to remove malicious nodes in time, and the multi-port could efficiently query the source and destination of products with legal benefits and accurately locate fault points.

\subsection{Solves the Problem of Traceability Trust for Agricultural Products}

\subsubsection{Reputation Evaluation Mechanism}

Reputation system is an effective method to solve trust problems. Malik et al. [10] proposed the trust management framework of supply chain application based on blockchain, assigned credit scores to participants and products, conducted qualitative security analysis on threats in the reputation system, and improved the credibility of products. 


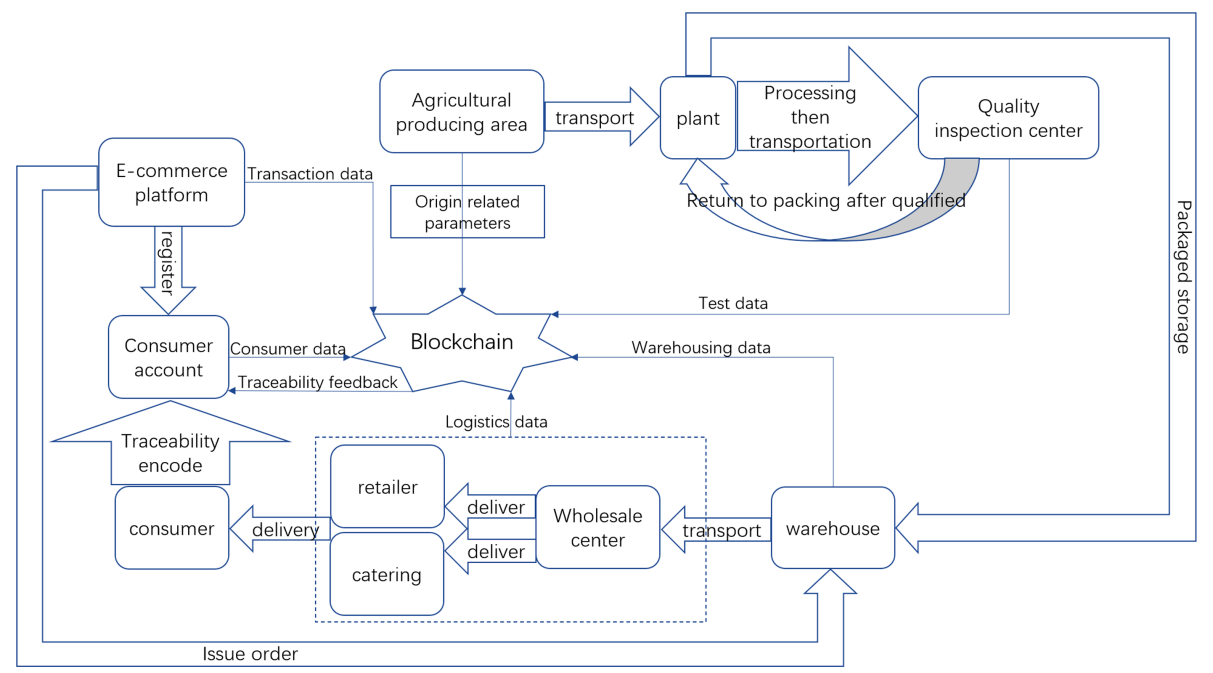

Fig. 2. Agricultural product traceability framework

\subsubsection{Reverse Technical Constraints}

When ethics cannot constrain the operational norms in agriculture, laws and technologies can be used for reverse restriction. The smart contract of blockchain forces users to fulfill the contract.

Pearson et al. [11] applied block chain to provide the encryption security of transactions and related metadata of the entire supply chain, including origin, contract, process steps, environmental changes, microbial records, etc., and the records are unchangeable, so as to meet the DLT requirements of data standardization in the field of agricultural products, and the whole product supply chain can be securely linked, to reverse the constraints of each link behavior.

\section{Summary and Prospect}

By discussing the current research papers, journals and projects related to blockchain in the agricultural field, it is found that the current research mainly focuses on supply chain, agricultural product traceability. In the research, most of them discuss the technical restrictions and loopholes related to blockchain, and a few of them restrict it morally and legally in combination with the regulatory system.

In the future, blockchain will have more applications in the agricultural field. From a technical perspective, the throughput of the system can be improved by optimizing the consensus algorithm, thus accelerating the upload process. Periodically clean expired information on the chain to reduce the accumulation of data volume; Strengthen data source management to improve the lack of credibility in the chain link; Update the encryption mechanism in time to increase the security of information; Collect data in the same way, unify data standards and improve data quality; Improve the privacy protection mechanism and increase the security of the member information on the chain. 
From the perspective of management, it can constantly improve the management mechanism in the field of agriculture and provide security guarantee. Increase the extension of rural finance and rural insurance to provide farmers with operational funds and security; improve the knowledge level of farmers, so that farmers can correctly understand the blockchain; reduce traceability costs, promote more product labeling information, etc.

Blockchain and agricultural applications need to be further integrated, and technology and management combined to improve the system. For example, smart contracts are combined with laws to limit the scope of contract execution, and contracts are used to confirm whether personnel follow the system, thus forming a two-way and mutually beneficial situation. The combination of blockchain and agriculture in the future remains to be explored by researchers.

\section{References}

1. Ren, M., Tang, H.B., You, W.: Survey of applications based on blockchain in government department. Comput. Sci. 45(02), 1-7 (2018). (in Chinese)

2. Patil, A.S., Tama, B.A., Park, Y., Rhee, K.-H.: A framework for blockchain based secure smart green house farming. In: Park, J.J., Loia, V., Yi, G., Sung, Y. (eds.) CUTE/CSA-2017. LNEE, vol. 474, pp. 1162-1167. Springer, Singapore (2018). https://doi.org/10.1007/978981-10-7605-3_185

3. Zhang, Q., Han, Y.Y., Su, Z.B., Fang, J.L., Liu, Z.Q., Wang, K.Y.: A storage architecture for high-throughput crop breeding data based on improved blockchain technology. Comput. Electron. Agric. 173(6) (2020). https://doi.org/10.1016/j.compag.2020.105395

4. Kim, H.M., Laskowski, M.: Agriculture on the blockchain: sustainable solutions for food, farmers, and financing. Social Science Electronic Publishing (2017)

5. Li, X.P., Wang, Y.Y.: Construction of logistics service supply chain information platform based on blockchain technology. Logistics Technol. 38(05), 101-106 (2019). (in Chinese)

6. Lucena, P., Binotto, A.P.D., Momo, F.S., Kim, H.M.: A case study for grain quality assurance tracking based on a blockchain business network. In: Symposium on Foundations and Applications of Blockchain (FAB 2018) (2018)

7. Huang, W., Chang, R.R., Chang, R.: The symbiotic development of rural e-commerce and logistics from the perspective of block chain tech. J. Commercial Econ. 6, 118-121 (2019). (in Chinese)

8. Huo, H., Zhan, S.: Construction of a whole-process supervision system for the quality and safety of agrifood from the perspective of integrated supply chain. Forum Sci. Technol. China 8, 105-113 (2019). (in chinese)

9. Liu, Z.M.: Research on "blockchain + RFID" enabling food traceability platform. Food Mach. 6, 1-8 (2020). (in Chinese)

10. Malik, S., Dedeoglu, V., Kanhere, S.S., Jurdak, R.: TrustChain: trust management in blockchain and IoT supported supply chains. In: IEEE International Conference on Blockchain. Semantic Scholar, Atlanta (2019). https://doi.org/10.1109/blockchain.2019. 00032

11. Pearson, S., May, D., Leontidis, G., Swainson, M., Brewer, S., Bidaut, L., et al.: Are distributed ledger technologies the panacea for food traceability? Glob. Food Secur. 20, 145-149 (2019). https://doi.org/10.1016/j.gfs.2019.02.002 
Open Access This chapter is licensed under the terms of the Creative Commons Attribution 4.0 International License (http://creativecommons.org/licenses/by/4.0/), which permits use, sharing, adaptation, distribution and reproduction in any medium or format, as long as you give appropriate credit to the original author(s) and the source, provide a link to the Creative Commons license and indicate if changes were made.

The images or other third party material in this chapter are included in the chapter's Creative Commons license, unless indicated otherwise in a credit line to the material. If material is not included in the chapter's Creative Commons license and your intended use is not permitted by statutory regulation or exceeds the permitted use, you will need to obtain permission directly from the copyright holder. 